\title{
Evaluating the Effect of Conservative Therapy in Patients with Wilkes Stage III Temporomandibular Joint Derangement
}

\section{Vladimír Machoň, Jitka Levorová, Michal Beňo, Dušan Hirjak, Milan Drahoš, René Foltán}

Department of Dental Medicine, First Faculty of Medicine, Charles University and General University Hospital in Prague, Prague, Czech Republic

Received February 22, 2021; Accepted October 20, 2021.

Key words: Temporomandibular joint conservative physiotherapy treatment

Abstract: The authors evaluated effects of physiotherapy in patients experiencing Wilkes III temporomandibular joint (TMJ) derangement with clinically limited joint mobility, but no pain. The group consisted of 31 patients with unilateral temporomandibular joint involvement, 3 men and 28 women (average age was 30.93 , ranging from age 12 to 61). None of the patients in the group had experienced any previous TMJ therapy. The patients underwent conservative therapy in the form of home exercise (mobilisation and isometric exercises) as the first step in treatment. The authors evaluated the improvement in jaw movement (maximal interincisal opening - MIO) and the patients' subjective assessments of their condition. Disc position before and after two months of exercise was also evaluated using ultrasound examination. The average MIO value in patients before starting the exercises was $33.5 \mathrm{~mm}$, and after two months of exercises, $42.4 \mathrm{~mm}$. Subjective assessment by patients: 26 patients (83\%) described their condition as completely satisfactory, not requiring further therapy. Of these patients, ultrasound examination showed 10 patients with complete disc reduction, 9 patients with a change in disc displacement with reduction, and 7 patients with a continuing (unchanged) state of disc displacement. Results of our study show the effect of conservative therapy in patients with painless TMJ due to disc displacement (WIII). Effect of home exercises which were easy to perform, simple and acceptable to the patient were demonstrated.

Mailing Address: Vladimír Machoň, MD., Department of Dental Medicine, First Faculty of Medicine, Charles University and General University Hospital in Prague, U Nemocnice 2, 12000 Prague 2, Czech Republic; e-mail: machonv@seznam.cz 


\section{Introduction}

Wilkes stage III (WIII) is a term for an intra-articular disorder of the temporomandibular joint (TMJ) which clinically manifests as limited jaw mobility, blocked jaw movement, frequent pain, joint tenderness and headache. WIII is characterised by disc displacement without reduction and thinning-deformation of the disc. Degenerative changes in the head of the joint and socket are not present (Wilkes, 1989). Treatment of WIII is usually conservative (non-surgical). If conservative treatment fails, minimally invasive procedures (arthrocentesis) or surgery (arthroscopic lavage and lysis, arthroscopic surgery, open surgery) are necessary. (Tenenbaum et al., 1999; Okeson, 2008; Machoň, 2017).

Physiotherapy is an integral part of conservative therapy for temporomandibular joint disorders (TMD), as is the use of occlusal splints or drug therapy. In the case of disc displacement without reduction, the main treatment option is physiotherapy with manual manipulation, especially in acute cases, and muscle massage, relaxation (in the presence of muscle spasm), muscle exercises (passive muscle stretching, assisted muscle stretching, resistance exercises) (Okeson, 2008).

The aim of the study was to evaluate the effect of physiotherapy on maximum opening of the mouth in patients with unilateral, painless WIII, who had not yet undergone any therapy. Another goal was to assess how many patients subjectively regarded their condition as satisfactory, not requiring further care after physiotherapy, and to assess disc position in these patients to see whether any change had occurred since the commencement of exercises. Another aim was to evaluate whether the average age of patients and average duration of the patient's symptoms contributed to any improvement in disc position.

\section{Material and Methods}

The primary treatment protocol for patients with WIII at the authors' workplace consists of educating the patient. The next step varies depending on the presence of pain. Patients who experience WIII without pain begin the next step in treatment with physiotherapy. If their condition does not improve within two months, the use of an occlusal splint follows, and physiotherapy is continued. In patients who experience WIII with pain present, treatment begins with the use of analgesics (NSAIDs), muscle relaxants and the application of thermotherapy. If pain reduces within 10 days, the patient begins physiotherapy. In the opposite case, arthrocentesis is indicated (Machoň, 2017).

Physiotherapy takes place in the form of home exercise, which consists of two types:

1) Extending the jaw forward (into protrusion), then opening the mouth and biting down (repeat three times, at least three times a day) (Figures 1-3);

2) Supporting the jaw with the palms, then attempting to open the mouth for 10 seconds, pushing against the palm. Relaxing the palm after 10 seconds and slowly opening the mouth (repeat three times, at least three times a day) (Figures 4 and 5). 

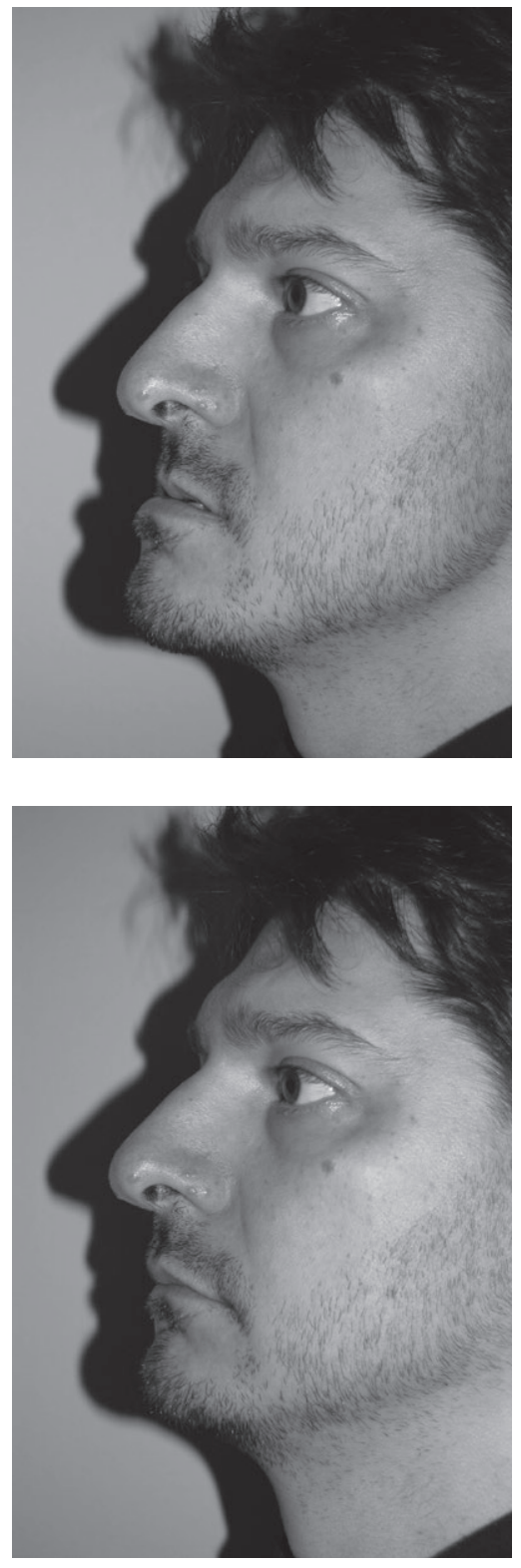

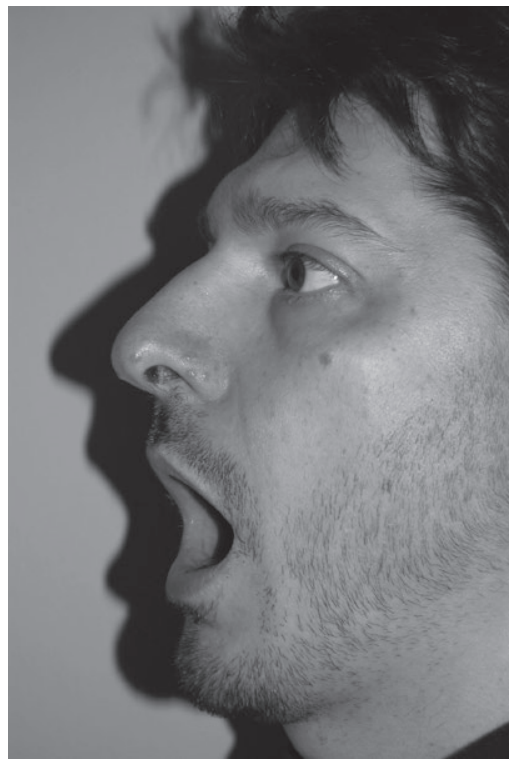

Figures 1-3 - Extending the jaw forward (into protrusion), then opening the mouth and biting down.

In addition to a clinical examination, all patients at the authors' workplace undergo an ultrasound examination of the joint (Mindray DP-50, 7.5 MHz, Shenzen Mindray Bio-medical Electronics) to determine a precise diagnosis. This examination is also performed on all patients six weeks after the commencement of exercise. This treatment protocol was also applied to the patients evaluated in the study. 

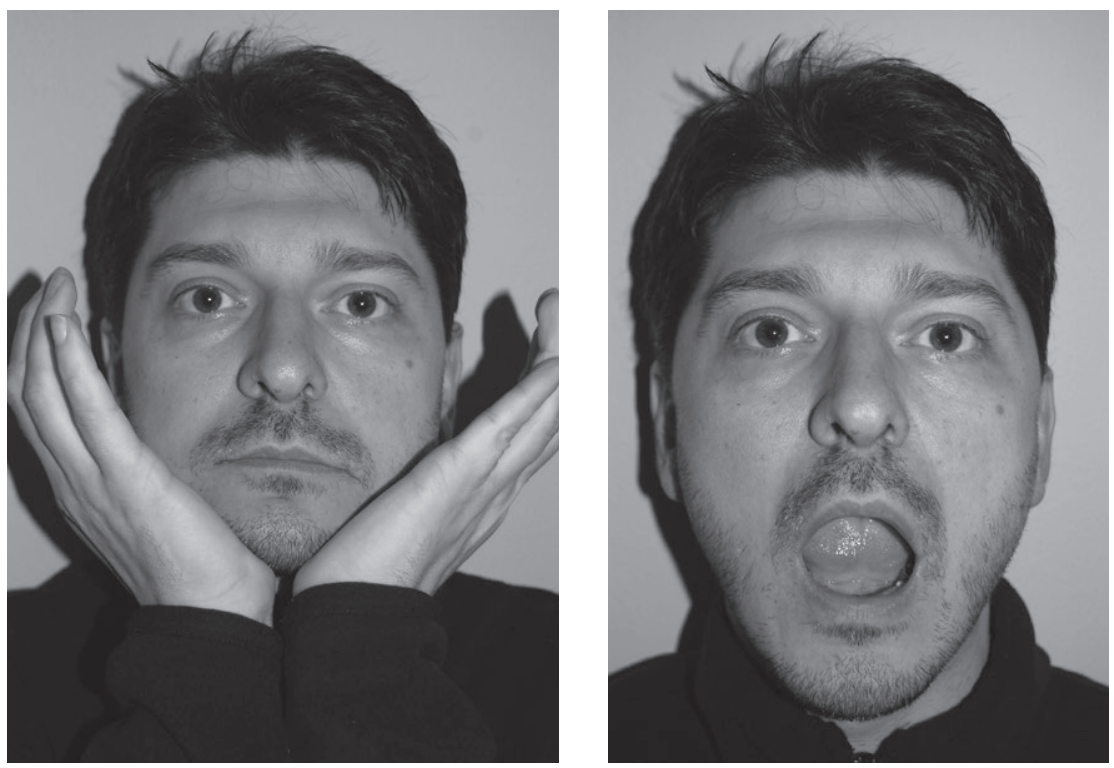

Figures 4 and 5 - Supporting the jaw with the palms, then attempting to open the mouth for 10 seconds, pushing against the palm. Relaxing the palm after 10 seconds and slowly opening the mouth.

Criteria for the selection of patients for the evaluation study:

Patients diagnosed with unilateral disc displacement without reduction, where patients experience limited mobility of the affected joint, although without any pain present. In addition, no patients have any systemic diseases (autoimmune, endocrinological, osteological). Patients who had already received some form of therapy were not included in the study. The study also excluded patients with palpable pain of the masticatory muscles, with the presence of muscle spasms, and patients who had been treated or were currently receiving physiotherapy treatment for problems associated with their neck muscles or cervical spine.

The authors examined 175 patients with blocked jaw movement due to disc displacement (WIII); however, only 31 had a unilateral, painless disorder which satisfied the study criteria, i.e. 3 men and 28 women (average age was 30.93, ranging from age 12 to 61 ).

Evaluation of the effect of exercise:

Maximum interincisal opening (MIO in millimetres), symmetry of movement and the presence of potential acoustic phenomena were noted in patients before the commencement of therapy.

If no deterioration in the condition was observed, the first follow-up examination was conducted two months after the commencement of treatment with home exercise, during which: 
a) MIO, symmetry of jaw movement, the presence of acoustic phenomena and the presence of any pain were clinically evaluated;

b) Patients were asked for their subjective view whether they felt an improvement in their condition, whether the state of their TMJ was such that it did not require further treatment, or conversely, whether it required the continuation of therapy.

Another factor was the evaluation of ultrasound findings, i.e. the position of the disc in patients before and two months after the commencement of exercises.

Statistical evaluation was applied to assess groups of patients who exhibited:

- Displaced disc reduction

- A change in disc displacement with reduction

following exercise, or who showed no change in disc position. Student's $t$-test was applied for evaluation and compared the age of patients and duration of symptoms. Statistically significant differences were set at $p<0.05$.

\section{Results}

Evaluation of patients before the commencement of home physiotherapy:

The average MIO value was $33.5 \mathrm{~mm}$, with jaw movement deviating to the affected side in all cases, while acoustic phenomena and pain were not recorded in any case. Ultrasound examination of all patients showed anterior disc displacement. The average duration of blocked movement before the commencement of treatment was 4.15 months (1-12 months).

During the two months of home exercise, no patient experienced a deterioration in condition which required monitoring sooner than two months after the commencement of therapy.

The average MIO value was $42.4 \mathrm{~mm}$. Improvement in opening of the mouth occurred in 25 patients, with an average improvement in movement of $9 \mathrm{~mm}$ (3-23 mm).

In 10 patients (32\%), lower jaw movement showed symmetry; 9 patients (29\%) recorded movement of the jaw with deflection to the side of the joint with disc displacement, while in 12 patients (39\%), movement with deviation to the affected side persisted.

Pain was not reported by any patient, and acoustic phenomena (clicking) were observed in 9 patients during jaw movement.

Patients' subjective assessments: 26 patients (83\%) described their condition as completely satisfactory, not requiring further therapy. According to ultrasound examination, 10 of these patients experienced complete disc reduction (38\% the average age was 28.9 , with an average duration of symptoms of 2.3 months). In 9 patients, a change in the state of disc displacement with reduction occurred (34\% - the average age was 32.7 , with an average duration of symptoms of 2.1 months). Permanent disc displacement persisted in 7 patients (22\% - the average age was 31.25 , with an average duration of symptoms of 6.1 months). 
Statistical evaluation did not show a statistically significant difference between the groups with complete disc reduction, disc displacement with reduction or persistent disc displacement without reduction following therapy in terms of the patients' average age $(P$-value $=0.29)$ and the duration of symptoms $(P$-value $=0.4068)$.

Five patients subjectively assessed their condition as unsatisfactory, requiring further treatment - these were all patients with persistent disc displacement without reduction according to the ultrasound examination.

\section{Discussion}

Conservative therapy for patients diagnosed with WIII is the basic treatment method and produces results comparable to mini-invasive and surgical therapy (Schiffman et al., 2007, 2014). Given that conservative therapy methods relating to joint structures are reversible, with minimal risk of structural damage, they are always the first treatment method selected (Randolph et al., 1990, Okeson, 2008). The goal of conservative therapy is to reduce pain (use of nonsteroidal anti-inflammatory drugs, soft food diet, application of heat) and subsequently improve opening of the mouth through physiotherapy and joint mobilisation (Nicolakis et al., 2001; Okeson, 2008; Machoň, 2017).

A basic step in physiotherapy in patients who experience WIII is manual mobilisation (MM), a manual disc reduction technique recommended by many authors as a first therapeutic step (Foster et al., 2000; Okeson, 2008; Miernik and Więckiewicz, 2015). Therefore, for successful MM, it is ideal if the condition is the patient's first episode of blocked joint movement and that the duration of symptoms has been less than one week (Okeson, 2008). Another factor for the success of therapy is the state of disc displacement. Changes and deformations reduce the success of MM (Kurita et al., 1999; Foster et al., 2000). For greater therapeutic effect, MM should be performed under general anaesthesia using central myorelaxation; however, this places greater stress on the patient (Kurita et al., 1999; Foster et al., 2000). The authors did not perform MM, mainly because most patients consult the authors' workplace after experiencing several weeks of symptoms (the average duration of blocked jaw movement in the study was 3.5 months).

In addition to joint mobilisation, physiotherapy also focuses on the involvement of masticatory muscles and the treatment of muscle co-contraction and muscle pain (Okeson, 2008). In the authors' work, the treatment of patients with painless WIII only focused on exercises which led to joint mobilisation; patients with evident muscle pain were not included in the study.

A number of exercise protocols are mentioned in the literature for patients with TMD. The Rocabado $6 \times 6$ programme is often mentioned (Rocabado and Iglarsh, 1991) (rest position of the tongue, control of TMJ rotation, rhythmic stabilisation technique, axial extension of the neck, shoulder posture, stabilised head flexion): these six exercises are performed six times a day. Kraus (1988) presents exercises in three parts. The first includes the rest position of the tongue, the relative position 
of the jaw, diaphragmatic breathing, palpation and strength training. The second part focuses on neuromuscular control of mandibular movement. The third part includes isometric exercises. Yoshida et al. $(2011,2013)$ recommend exercises in two stages for patients with WIII; the first stage of the exercise programme consists of active movements repeated for 10 minutes, i.e. lateral movement towards to the right and left, protrusion and opening of the mouth. The second stage consists of passive exercises, i.e. opening the mouth with the fingers. Both stages are repeated at least eight times per day.

The authors are convinced that exercises performed by the patient at home are especially important for successful physiotherapy (Yoshida et al., 2011, 2013; Shafferr et al., 2014). Physiotherapy performed under repeated supervision by a therapist or physician can be limiting for many patients in terms of time. Another factor contributing to success is the simplicity of the exercises and their ease of performance (Yoshida et al., 2011, 2013; Shaffer et al., 2014). Therefore, the authors of the study recommend only two types of exercises for patients: mobilisation exercises (where the patient moves their jaw into protrusion, opening the mouth) and isometric exercises. The authors believe that a greater number of exercises becomes a less attractive option due to the time required to perform the exercises for some patients and the routine being less clear and comprehensible to others. Fewer exercises are easier for the patient to master to achieve regular and correct performance.

In addition to the performance of physiotherapy, good communication between the physician and patient, thorough instruction and patient motivation are also important in treatment. The physician's initial consultation with the patient should include a careful explanation of the patient's problem, the possible causes and treatment options (Randolph et al., 1990). The authors emphasise the need for thorough instruction and practice of the exercises.

The results of the authors' study show that $83 \%$ of patients subjectively viewed the therapy as effective and were satisfied with their condition after two months, characterising it as requiring no further therapy. Objective evaluation of jaw movement showed an improvement in opening of the mouth in $80 \%$ of patients, with an average improvement in movement of $9 \mathrm{~mm}(3-23 \mathrm{~mm})$. These results are comparable to the work of other authors (Nicolakis et al., 2001; Shiffman et al., 2007, 2014).

Ultrasound examination showed an objective improvement in condition in 19 patients (61\%) after exercise: in 10 cases, complete disc reduction occurred (32\%), and in 9 cases, disc displacement with reduction when opening the mouth was seen (29\%). Improved disc mobility was seen in patients with an average duration of symptoms of 2.2 months, while in patients where disc mobility did not improve, the average duration of symptoms was 6.1 months. This may confirm the fact that conservative therapy and physiotherapy are more effective in patients with acute symptoms (Randolph et al., 1990; Miernik and Więckiewicz, 2015). However, 
this was not verified by the statistical evaluation in the authors' study. Furthermore, spontaneous adjustment of disc position must not be disregarded in the evaluation of the effect of therapy (Sato et al., 1997; Shiffman et al., 2014).

The authors' study has several disadvantages. This is a simple evaluation of therapy without a comparison group; therefore, the placebo effect of these exercises cannot be clearly assessed. Another negative aspect is the low number of patients (31 patients), which was affected by the conditions for the inclusion of patients in the study. The third negative factor is the evaluation of disc position using ultrasound examination alone, whereas much greater accuracy is achieved with magnetic resonance imaging (Almeida et al., 2019). On the other hand, ultrasound examination is readily available (waiting time for magnetic resonance imaging of the TMJ is up to 6 weeks at the authors' workplace) and may be performed immediately, with a clear advantage from an economic perspective.

\section{Conclusion}

Results of our study show the effect of conservative therapy in patients with painless blocked movement of the TMJ due to disc displacement (WIII). The authors demonstrated the effect of simple, easy to perform home exercises which were acceptable to the patient. However, the integral part of the success of treatment is the patient's motivation and instruction, and, of course, the effect of spontaneous disc adjustment.

\section{References}

Almeida, F. T., Pacheco-Pereira, C., Flores-Mir, C., Le, L. H., Jaremko, J. L., Major, P. W. (2019) Diagnostic ultrasound assessment of temporomandibular joints: A systematic review and meta-analysis. Dentomaxillofac. Radiol. 48, 2-16.

Foster, M. E., Gray, R. J., Davies, S. J., Macfarlane, T. V. (2000) Therapeutic manipulation of the temporomandibular joint. Br. J. Oral Maxillofac. Surg. 38, 641-644.

Kraus, S. L. (1988) TMJ Disorders: Management of the Craniomandibular Complex. Churchill Livingstone, New York.

Kurita, H., Kurashina, K., Ohtsuka, A. (1999) Efficacy of a mandibular manipulation technique in reducing the permanently displaced temporomandibular joint disc. J. Oral Maxillofac. Surg. 57, 784-787.

Machoň, V. (2017) Manual of TMJ Surgery. The Prague Approach. Business Media Praha, Praha.

Miernik, M., Więckiewicz, W. (2015) The basic conservative treatment of temporomandibular joint anterior disc displacement without reduction - Review. Adv. Clin. Exp. Med. 24, 731-735.

Nicolakis, P., Erdogmus, B., Kopf, A., Ebenbichler, G., Kollmitzer, J., Piehslinger, E., Fialka-Moser, V. (2001) Effectiveness of exercise therapy in patients with internal derangement of the temporomandibular joint. J. Oral Rehabil. 28, 1158-1164.

Okeson, J. P. (2008) Management of Temporomandibular Disorders and Occlusion, $6^{\text {th }}$ Edition. Mosby Elsevier, St. Louis.

Randolph, C. S., Greene, C. S., Moretti, R., Forbes, D., Perry, H. T. (1990) Conservative management of temporomandibular disorders: A posttreatment comparison between patients from a university clinic and from private practice. Am. J. Orthod. Dentofacial Orthop. 98, 77-82.

Rocabado, M., Iglarsh, Z. A. (1991) Musculoskeletal Approach to Maxillofacial Pain. J. B. Lippincott, Philadelphia. 
Sato, S., Kawamura, H., Nagasaka, H., Motegi, K. (1997) The natural course of anterior disc displacement without reduction in the temporomandibular joint: follow-up at 6-, 12-, and 18-months. J. Oral Maxillofac. Surg. 55, 234-239.

Schiffman, E. L., Look, J. O., Hodges, J. S., Swift, J. Q., Decker, K. L., Hathaway, K. M., Templeton, R. B., Fricton, J. R. (2007) Randomized effectiveness study of four therapeutic strategies for TMJ closed lock. J. Dent. Res. 86, 58-63.

Schiffman, E. L., Velly, A. M., Look, J. O., Hodges, J. S., Swift, J. Q., Decker, K. L., Anderson, Q. N., Templeton, R. B., Lenton, P. A., Kang, W., Fricton, J. R. (2014) Effects of four treatment strategies for temporomandibular joint closed lock. Int. J. Oral Maxillofac. Surg. 43, 217-226.

Shaffer, S. M., Brismée, J. M., Sizer, P. S., Courtney, C. A. (2014) Temporomandibular disorders. Part 2: Conservative management. J. Man. Manip. Ther. 22, 13-23.

Tenenbaum, H. C., Freeman, B. V., Psutka, D. J., Baker, G. I. (1999) Temporomandibular disorders: Disc displacements. J. Orofac. Pain 13, 285-290.

Wilkes, C. H. (1989) Internal derangement of the temporomandibular joint: Pathological variations. Arch. Otolaryngol. Head Neck Surg. 115, 469-477.

Yoshida, H., Sakata, T., Hayashi, T., Shirao, K., Oshiro, N., Morita, S. (2011) Evaluation of mandibular condylar movement exercise for patients with internal derangement of the temporomandibular joint on initial presentation. Br. J. Oral Maxillofac. Surg. 49, 310-313.

Yoshida, H., Kashiwagi, K., Sakata, T., Tanaka, M., Kawazoe, T., Morita, S. (2013) Prognostic factor of mandibular condylar movement exercise for patients with internal derangement of the temporomandibular joint on initial presentation: Preliminary report. J. Craniomanillofac. Surg. 41, 356-358. 